\title{
Arterial compliance: a tool in the evaluation of vascular disease in familial hypercholesterolemia
}

\author{
Pedro Marques da Silva
}

Hypertension and Dyslipidaemias Unit, Department of Internal Medicine, Hospital de Santa Marta, Rua de Santa Marta, 1150 Lisboa, Portugal

\section{Introduction}

Until recently, clinicians concerned about diastolic blood pressure and peripheral vascular resistance have tended to suppress the importance of the systolic blood pressure and of arterial stiffness. However, increased peripheral vascular resistance, resulting from a reduction in the calibre and/or number of small arteries and arterioles, is only one characteristic of the functional components of the arterial system and does not represent an adequate description of the vascular impedance load $[1,2]$.

Some of the most frequent clinical problems in cardiology, such as hypertension and atherosclerosis, result from derangement of vascular mechanics. For the clinician, however, it is hard to understand the more complex concepts of haemodynamics and the mechanical principles of arterial disease [3,4].

\section{Vascular mechanics}

From a conceptual and physiological point of view, arteries have two different functions that are tightly related [5-7]. One, the conduit function, describes the capacity of the arterial system to deliver an adequate supply of blood from the heart to body tissues. To maintain this supply, the blood flow (and the mean blood pressure) must be steady and high enough to overcome any significant peripheral resistance. The other, the cushioning function of arteries (see Fig. 1), results from the capacity of large arteries to buffer intermittent pulsatile output and accommodate the blood volume ejected in systole, as well as draining this volume in a continuous steady flow into smaller arteries during diastole. This property ('Windkessel' or reservoir effect) is closely related to the viscoelastic properties of arterial walls and may be described in terms of compliance or distensibility.
In physiology, compliance $(C)$ is defined as the absolute diameter (or area) change of an artery for a given pressure step at a fixed vessel length $-\mathrm{d} D / \mathrm{d} P(\mathrm{~cm} /$ $\mathrm{mmHg}$ or $\mathrm{cm}^{2} / \mathrm{mmHg}$ ). Distensibility (Di) is the inverse of elastic modulus $-\mathrm{d} D / \mathrm{d} P \times D\left(\mathrm{mmHg}^{-1}\right)-$ and is the measure of relative arterial compliance that correlates the relative change in diameter to the imposed pressure step.

Many other terms may be used to quantify the elastic properties of the arteries [4]. All of them are site specific, depending on the intrinsic characteristics of the arteries and on the mean pressure at which they are calculated. In the same artery, they may also depend on the distending pressures to which they are subjected and on the structure and functional status of vascular smooth muscle of arterial media [2]. They may be calculated, directly or indirectly, from the evaluation of arterial pressure, diameter, flow, and wall thickness [1].

In the last few years, the development of non-invasive techniques of ultrasonography and aplanation tonomery (sphygmocardiography) has allowed a renewed interest in the study of arterial properties [7].

As a consequence of two different functions of arteries, the arterial pressure has two components: one, the steady component, represented by mean blood pressure; and the other, the pulsatile component, related to the oscillation around mean blood pressure and represented by pulse pressure. The mean blood pressure depends on cardiac output and on the cross-sectional area and number or arterioles and small arteries. The pulse pressure, in turn, depends on the characteristics of left ventricular ejection, on arterial stiffness, and on the timing of arterial reflection.

There is another important haemodynamic concept to remember: The reflection of the arterial wave is a natural consequence of vascular design $[2,8]$. The proximal aorta and its major branches behave as long, highly compliant, arterial vessels that terminate abruptly in small, less compliant, distal vessels. These distal vessels are very important to circulatory regula- 


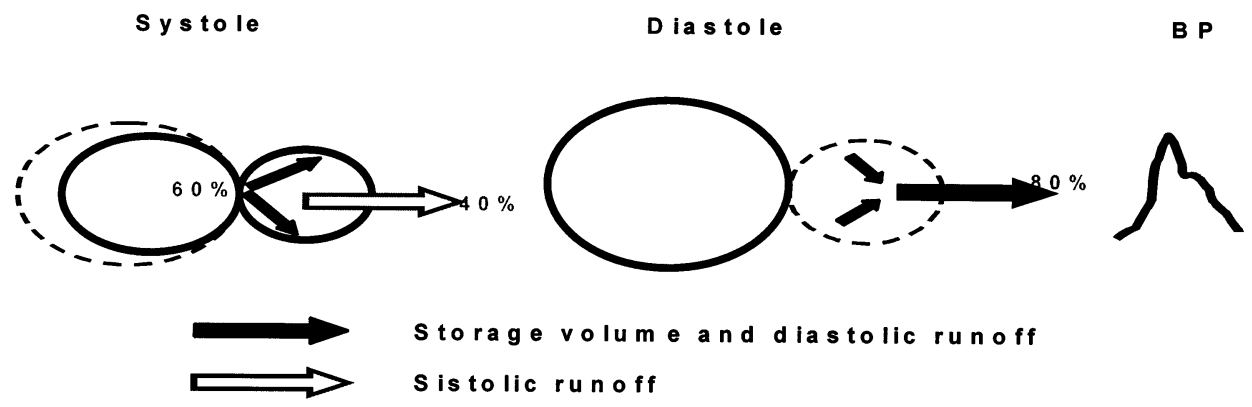

Fig. 1. The cushioning function of arteries.

tion because they account for the generation of an echo (reflected) wave that travels back towards the heart.

In healthy young people with high aortic compliance, distensible arteries, and low pulse wave velocity (PWV), the wave reflection occurs late during early diastole and after ventricular ejection has stopped. This is physiologically advantageous to maintain a normal cardiac function and coronary perfusion pressure during all of diastole.

Pulse wave velocity $(a)$, the speed of travel of the pulse along the arterial segment — distance $/ t(\mathrm{~cm} / \mathrm{s})-$ depends on the arterial stiffness and determines how quickly the reflected wave echo returns [8]. So, when there is an increase in PWV or shortening in the effective arterial length, there is a consequent early return of wave reflection from the periphery to the heart. This early return boosts the aortic (and ventricular) pressure in late systole; increases mean-, peak-, and end-systolic blood pressure; and reduces the mean aortic pressure during diastole. On the other hand, myocardial work load and oxygen consumption increases.

Finally, increases in systolic blood pressure and pulse pressure intensify degenerative changes, arterial damage, and arterial stiffening, thus contributing to the establishment of a deleterious vicious cycle [2].

The decrease in arterial compliance may, therefore, be a passive consequence of an increase in blood pressure - reduced arterial compliance is frequently accepted as a feature of hypertension - or the result of structural and/or functional alterations in the arterial wall that may occur in several metabolic diseases. However, in metabolic conditions frequently associated with hypertension (obesity, diabetes, insulin resistance and hyperlipidemias), the data on possible changes in haemodynamics parameters are scarce and frequently controversial [9].

\section{Arterial compliance and atherosclerosis}

Cameron et al. [10], tried to study the effect of serum lipids on systemic arterial compliance in a group of patients with newly diagnosed coronary artery disease (meaning that they had not started modifications of their lifestyle or dietary habits) and in matched controls. They showed a significant negative correlation between arterial compliance and low-density lipoprotein (LDL)-cholesterol blood levels. In patients with coronary disease, there was more severe change in aortic mechanical properties than in disease-free subjects, despite identical lipid plasma levels in the two groups. Other authors have also shown the importance of the amount and severity of coronary atherosclerosis for the expression of a more generalised disease in the aorta and in its major branches — with the expected consequences in the viscoelastic properties of larger arteries. Barenbrock et al. [11], demonstrated that arterial distensibility was markedly affected only in patients with severe atherosclerotic disease. Patients with moderate hypercholesterolemia, with no evidence of clinical atherosclerosis, seem to have a low risk of developing impaired arterial compliance.

However, recently, Giannattasio et al. [12], showed that isobaric compliance and distensibility of middlesized conduit arteries were significantly reduced in hypertensive patients with mild hypercholesterolemia and in non-hypertensive hypercholesterolemic patients. The same authors have found that heterozygous familial hypercholesterolemia $(\mathrm{FH})$ is associated with a striking reduction $(-53.5 \% ; P<0.01)$ in radial artery compliance compared to normotensive normocholestemlemic healthy subjects [13].

Surprisingly, in hypercholesterolemic patients with no apparent genetic lipid abnormality, the arterial compliance was similar to normocholesterolemic controls [14]. Apparently, there is no explanation for this difference. The authors suggest the existence of a factor that disturbs normal endothelial function or modifies the normal structure and the viscoelastics properties of the arterial wall. We can speculate that this difference in the arterial compliance might be related to the coexistence of other eventual genetic factors or with potential gene-gene interactions.

It is important to remember that genetic and experimental studies have related the renin-angiotensin system and angiotensin-converting enzyme (ACE)/ 
angiotensin II type I (AT1) receptor polymorphisms to large-artery damage, aortic stiffness and changes in extracellular matrix $[9,15]$. O'Malley et al. [16], showed that ACE DD genotype is associated with an increased risk for premature development of coronary atherosclerosis and myocardial infarction in patients with heterozygous FH. These investigators have not pondered the possibility that a change in vascular haemodynamics might contribute to an increase in risk. This certainly will be one of the fields worth further investigation.

Some studies are less explicit about the effect of $\mathrm{FH}$, hypercholesterolemia, and atherosclerosis in the arterial compliance. Others present conflicting results on the effects of hyperlipidemia and other major cardiovascular risk factors in vascular haemodynamics. Atherosclerosis, in contrast to arteriosclerosis (stiffening and dilatation of major arteries), is normally accepted as an example of a focal vascular disease that predominantly affects conduit function. However, mechanical stress in the arterial wall may be an important element in atherogenesis and in the development of atherosclerotic plaque. It is also involved in plaque rupture. Further, the type of blood flow is an important determinant for the organisation of thrombus [17].

Pathologically, the atherosclerotic changes of the arterial wall include an increase in wall thickness and deposition of lipids (with xanthomatous lesions in $\mathrm{FH}$ ) in the intima, smooth muscle cell (SMC) proliferation with macrophage- and SMC-derived foam cell formation (presence of extensive fat vacuolation of SMC in $\mathrm{FH}$ ), as well as excessive synthesis and accumulation of fibrillary and non fibrillary connective tissue elements (collagen, elastin and proteoglycans) [18,19].

\section{Discussion}

All these morphological changes can modify the viscoelastic properties and the buffering capacity of larger arteries, but their importance may depend on the stage of the disease. In the early stages of atherosclerosis in animals where there is a greater accumulation of cholesterol and foam cells in the intima, the aorta is more distensible than normal, only to stiffen later when collagen and calcium predominate in the arterial wall [20].

Hopefully, long term aggressive LDL-cholesterol reduction with statins in patients with polygenic hypercholesterolemia and heterozygous FH with or without coronary heart disease, can slow the development of atherosclerosis and improve the progressive stiffness of larger arteries with potential benefits in cardiovascular haemodynamics and in cardiac function [13,21-23].

The data on this subject are limited. Explanations and extrapolations must be made with caution. How- ever, current non-invasive modern technology for the analysis of arterial compliance and PWV may potentially be a useful research tool in the study of patients with familial hypercholesterolemia and a greater susceptibility to atheroma.

\section{References}

[1] McVeigh GE, Finkelstein SM, Cohn JN. Assessment of arterial compliance in hypertension. Curr Opin Nephrol Hypertens $1993 ; 2: 82-6$.

[2] London GM. Large artery function and alterations in hypertension. J Hypertens 1995;13(Suppl 2):S35-8.

[3] Lee RT, Kamm RD. Vascular mechanics for the cardiologist. J Am Coll Cardiol 1994;23:1289-95.

[4] O'Rourke MF. Mechanical principles in arterial disease. Hypertension 1995;26:2-9.

[5] Marchais SJ, Guerin AP, Pannier B, Delavaud G, London GM. Arterial compliance and blood pressure. Drugs 1993;46(Suppl 2):82-7.

[6] London GM. Large arteries haemodynamics: conduit versus cushioning function. Blood Press 1997;6(Suppl 2):48-51.

[7] Nichols WW, O'Rourke MF. McDonald's Blood Flow in Arteries. Theoretical, experimental and clinical principles, 4th ed. London: Edward Arnols Publishers, Ltd, 1998.

[8] O'Rourke MF. Arterial compliance and wave reflection. Arch Mal Cœur 1991;84(III):45-8.

[9] Safar ME, Frohlich ED. The arterial system in hypertension. A prospective view. Hypertension 1995;26:10-4.

[10] Cameron JD, Jennings GL, Dart AM. The relationship between arterial compliance, age, blood pressure and serum lipid levels. J Hypertens 1995;13:1718-23.

[11] Barenbrock M, Spieker C, Kerber S, Vielhauer C, Hoeks APG, Zidek W, Rahn K-H. Different effects of hypertension, atherosclerosis and hyperlipidaemia on arterial distensibility. J Hypertens 1995;13:1712-7.

[12] Giannattasio C, Mangoni AA, Failla M, Stella ML, Carugo S, Bombelli M, Sega R, Mancia G. Combined effects of hypertension and hypercholesterolemia on radial artery function. Hypertension 1997;29:583-6.

[13] Giannattasio C, Mangoni AA, Failla M, Carugo S, Stella ML, Stefanoni P, Garssi O, Vergani C, Mancia G. Impaired radial artery compliance in normotensive subjects with familial hypercholesterolemia. Atherosclerosis 1996;124:249-60.

[14] Giannattaslo C, Mangoni AA, Carugo S, Bombelli M, Stefanoni P, Failla M, Stella ML, Sega R, Garssi G, Vergani C, Mancia G. Arterial compliance in familial hypercholesterolemia: a preliminary report. J Hypertens 1993;11(Suppl 5):S82-3.

[15] Benetos A, Topouchian J, Ricard S, Gautier S, Bonnardeaux A, Asmar R, Piorier O, Soubrier F, Safar M, Cambien F. Influence of angiotensin type 1 receptor polymorphism on aortic stiffness in never-treated hypertensive patients. Hypertension 1995;26:447.

[16] O'Malley JP, Maslen CL, llingworth R. Angiotensin-converting enzyme DD genotype and cardiovascular disease in heterozygous familial hypercholesterolemia. Circulation 1998;97:1780-3.

[17] Texon M. Hemodynamic Basis of Atherosclerosis, 2nd ed. New York: Begell House, Inc, 1995.

[18] Stehbens WE, Martin M. The vascular pathology of familial hypercholesterolemia. Pathology 1991;23:54-61.

[19] Schwartz CJ, Chandler AB. Coronary atherosclerotic disease: pathological background. In: Fuster V, editor. Syndromes' of Atherosclerosis: Correlations of Clinical Imaging and Pathology, 1st edition. Armonk, NY: Futura Publishing Company, Inc, 1996:17-32. 
[20] Kupari M, Hekali P, Keto P, Poutanen V-P, Tikkanen MJ. Standertskjöld-Nordenstam. Relation of aortic stiffness to factors modifying the risk of atherosclerosis in healthy people. Arterioscler Thromb 1994;14:386-94.

[21] Forbat SM, Naoumova RP, Sidhu PS, Neuwirth C, MacMahon M, Thompson GR, Underwood SR. The effect of cholesterol reduction with fluvastatin on aortic compliance, coronary calcification and carotid intimal-medial thickness: a pilot study. J Cardiovasc Risk 1998;5:1-10.
[22] Tomochika Y, Okuda F, Tanaka N, Wasaki Y, Tokisawa I, Aoyagi S, Morikuni C, Ono S, Okada K, Matsuzaki M. Improvement of atherosclerosis and stiffness of the thoracic descending aorta with cholesterol-lowering therapies in familial hypercholesterolemia. Arterioscler Thromb Vasc Biol 1996;16:955-62.

[23] Wilkinson IB, Cockcroft JR. Cholesterol, endothelial function and cardiovascular disease. Curr Opin Lipidol 1998;9:23742. 